\title{
CARBON AND NITROGEN IN THE COOL SUPERGIANT A7 OF THE YOUNG GLOBULAR CLUSTER NGC 330 IN THE SMC*
}

\author{
B. BARBUY, A. MILONE \\ Universidade de Sao Paulo, Depto. Astronomia \\ CP30627 Sao Paulo 01051 \\ Brazil \\ M. SPITE, F. SPITE \\ Observatoire de Paris, Section d' Astrophysique \\ 92195 Meudon Pl Cedex \\ France
}

\begin{abstract}
C_{2}$ and $C N$ bands in the wavelength region $\lambda 560-680 \mathrm{~nm}$ were used to obtain the carbon and nitrogen abundances in the supergiant star A7 of the young, metal-poor, globular cluster NGC 330 in the Small Magellanic Cloud (SMC).
\end{abstract}

\section{Introduction}

NGC 330 is a young cluster (age $1.2 \times 10^{7}$ yrs (Carney et al. 1985)) in the SMC, showing a low metal abundance, $[\mathrm{Fe} / \mathrm{H}] \approx-1$ (where the standard notation $[\mathrm{X}]=\log \mathrm{X} * / \mathrm{X}_{\odot}$ is used). Spite et al. (1986) and Richtler et al. (1990) have made a detailed analysis of the red supergiant star A7 of this cluster, to determine its overall metal abundance, as well as the relative abundances of the other elements. They conclude that NGC 330:A7 shows elemental ratios characteristic of an old population cluster. It has been found for the $\mathrm{F}$ field supergiants that the abundance of carbon is "normal" (i.e. that the ratio C/Fe is the same in the Magellanic Clouds as in the Galaxy, see for example Spite and Spite (1990), whereas in the HII regions the carbon abundance seems to be low by about 0.6 dex.

In the present work, we derive the carbon (C) and nitrogen $(\mathrm{N})$ abundances in the cool supergiant A7 of NGC330, which should reflect another step of the chemical evolution of the Clouds (i.e. as a sample of little evolved material). To our knowledge, this is the first detailed determination of the $\mathrm{C}$ and $\mathrm{N}$ abundances in the Clouds from molecular bands.

\section{Observations}

High resolution spectra in the wavelength region $\lambda 560-680 \mathrm{~nm}$ were obtained at the $3.6 \mathrm{~m}$ telescope of the European Southern Observatory (ESO), La Silla, Chile, using the échelle spectrograph CASPEC and a RCA CCD of 512x512 pixels.

\footnotetext{
* Observations at the European Southern Observatory ESO (Chile)
} 


\section{Calculations}

Synthetic spectra calculations were made in the regions containing bandheads of $\mathrm{C}_{2}$ and $\mathrm{CN}$ molecular lines. These bandheads correspond to the vibrational transitions $\left(v^{\prime}, v^{\prime \prime}\right)=(0,1),(3,5)$, $(2,5),(5,8)$ and $(1,4)$ of the $C_{2} A^{3} \Pi-X^{3} \Pi$ Swan system, and the transitions $(8,3),(5,1)$ and $(8,4)$ of the $\mathrm{CN} \mathrm{A}^{2} \Pi-\mathrm{X}^{2} \Sigma$ red system. In Figure 1 the $(2,5) C_{2}$ band is shown. The stellar parameters employed are those given by Richtler et al. (1990): $\left(\mathrm{T}_{\mathrm{eff}}, \log g,[\mathrm{M} / \mathrm{H}], \mathrm{v}_{\mathrm{t}}\right)=(3900$, $\left.-0.2,-1.05,4.5 \mathrm{~km} \mathrm{~s}^{-1}\right)$ or $(4000,0.0,-1.0,4.5)$ corresponding to reddenings $\mathrm{E}(B-V)=0.03$ and 0.12 respectively; the model atmosphere was obtained by an interpolation in the grid of models by Gustafsson et al. (1975).

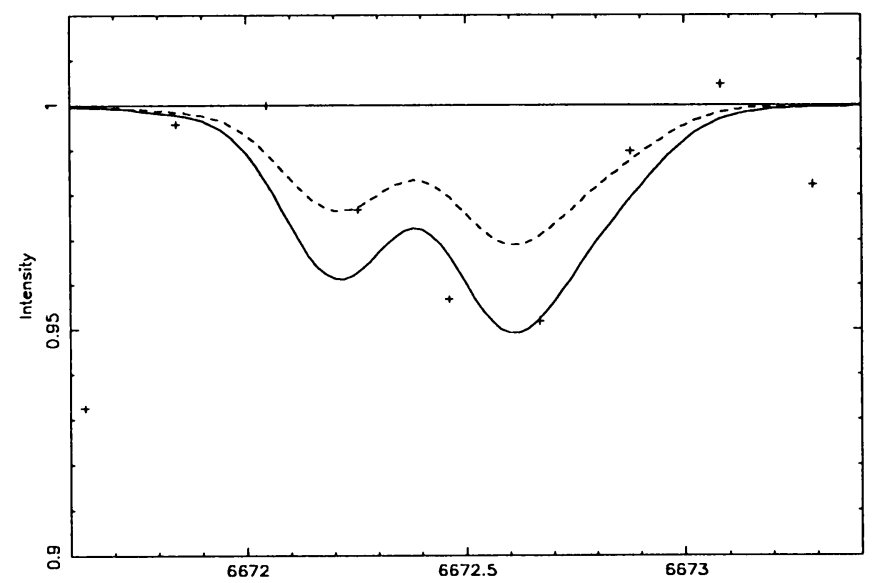

Figure 1. Observed spectra (crosses) and synthetic spectra of $\mathrm{C}_{2}(2,5)$ band for $[\mathrm{C} / \mathrm{Fe}]=+0.1$ (dashed line) and $[\mathrm{C} / \mathrm{Fe}]=+0.2$ (full line). The best fit was obtained for $[\mathrm{C} / \mathrm{Fe}] \approx 0.15$.

\section{Results and conclusions}

The best fits were found for $[\mathrm{C} / \mathrm{Fe}] \approx 0.15$ and $[\mathrm{N} / \mathrm{Fe}] \approx 0.0$, and therefore no carbon underabundance is seen, in disagreement with data from HII regions. The same result has already been found for field SMC stars by Spite et al. (1989), Russell \& Bessell (1989) and Spite \& Spite (1990).

\section{References}

Carney, B.W., Janes, K.A., Flower, P.J. (1985), Astron. J. 90, 1196.

Gustafsson, B., Bell, R.A., Eriksson, K., Nordlund, A. (1975), Astron. Astrophys. 42, 407.

Richtler, T., Spite, M., Spite, F. (1990), these proceedings.

Russell, S.C., Bessell, M.S. (1989), Astrophys. J. Suppl. 70, 865.

Spite, M., Spite, F. (1990), these proceedings.

Spite, M., Barbuy, B., Spite, F. (1989), Astron. Astrophys. 222, 35

Spite, M., Cayrel, R., François, P., Richtler, T., Spite, F. (1986), Astron. Astrophys. 168, 197. 